\title{
Filamentation Time Diagnosis of Thinning Troughs and Cutoff Lows
}

\author{
Yu-Ming Tsai* \\ Hung-Chi Kuo \\ Department of Atmospheric Sciences \\ National Taiwan University \\ Taipei, Taiwan \\ Wayne H. Schubert \\ Department of Atmospheric Science \\ Colorado State University \\ Fort Collins, Colorado
}

Submitted to Monthly Weather Review

17 June 2009

Revised 16 October 2009

Revised 4 December 2009

Accepted 17 January 2010

${ }^{*}$ Corresponding author address: Y.-M. Tsai, Department of Atmospheric Sciences, National Taiwan University, Taipei 106, Taiwan. E-mail: d95229003@ntu.edu.tw 


\begin{abstract}
This paper analyzes synoptic-scale trough thinning processes using a filamentation time diagnostic. The filamentation time diagnostic is derived from the potential vorticity equation expressed in spherical coordinates in the horizontal and the isentropic coordinate in the vertical. The diagnostic is an accurate measure of stirring processes under the condition of "slowly varying velocity gradients." Trough thinning processes are analyzed for one tropical example and two midlatitude examples. Our results indicate that the filamentation time for the tropical trough thinning event is generally longer than those for the midlatitude trough events. For the calculation of filamentation time on isentropic surfaces in spherical coordinates, it is acceptable to ignore the curvature effects in the tropics, but in both the midlatitudes and the tropics, isentropic divergence effects should be retained for improved accuracy. In addition to the potential vorticity on an isentropic surface, the filamentation time diagnostic contains the effects of divergence, stretching and shearing deformations. Combining the cross potential vorticity contour flows on isentropic surfaces with the filamentation time analysis gives a more complete description of the dynamics. The results show that the filamentation time diagnostic can serve as a useful aid in the analysis and prediction of trough thinning and cutoff low formation.
\end{abstract}




\section{Introduction}

A common phenomenon in mesoscale and synoptic scale atmospheric flows is the production of banded or filamentary structures in the potential vorticity (PV) field on isentropic surfaces. A simple physical reason for this is that stratification and background rotation constrain the flow to be quasi-two-dimensional, so that it roughly resembles idealized two-dimensional turbulence in which there is a selective decay of enstrophy over energy (McWilliams 1984). This selective decay process is associated with vortex mergers within a sea of filamentary structures produced by earlier vortex interactions. At the mesoscale, spiral rain bands and their associated PV structures are one of the most noticeable features associated with tropical cyclones (Guinn and Schubert 1993). At the synoptic scale, trough thinning phenomena are common in midlatitude baroclinic waves (Keyser and Shapiro 1986; Thorncroft et al. 1993, hereafter T93; Appenzeller et al. 1996; Kunz et al. 2009) and in tropical flows such as tropical upper tropospheric troughs (TUTTs, as discussed by Ferreira and Schubert 1999). T93 used a simple argument with "PV thinking" to propose two paradigms of baroclinic-wave lifecycle behaviour: (i) Troughs will thin if they are located in the anti-cyclonic shear on the equatorward side of the jet; (ii) Troughs will broaden if they are located in the cyclonic shear on the poleward side of the jet. In addition, the equatorward movement of high potential vorticity stratospheric air along "Middleworld" (Hoskins 1991) isentropes will extend troughs into the midlatitude upper troposphere and often lead to the formation of cutoff lows, also known as cold core lows. Readers who are interested in cold core lows are referred to Kelley and Mock (1982) for a synoptic scale composite study. Because filamentary structures are so common in atmospheric flows, it is interesting and useful to develop diagnostic tools to better understand the relevant formation processes.

The filamentation process is accompanied by an increase in the overall vorticity (or PV) gradient. This process can be quantified in a simple manner using criteria developed by Okubo (1970), Weiss (1991), and Koh and Legras (2002). In particular, Okubo studied the 
horizontal dispersion of floatable particles through an analysis of tracer gradient evolution, while Weiss derived the vorticity gradient growth rate from the barotropic vorticity equation, and Koh and Legras (2002) used hyperbolic lines of the strain deformation to analyse the evolution of the stratospheric polar vortex. In related work directed toward improved understanding of hurricane dynamics, Rozoff et al. (2006, hereafter R06) defined a local parameter called the "filamentation time," which is the e-folding time for growth of the vorticity gradient. R06 argued that the formation of a moat region between hurricane concentric eyewalls may be partially caused by the strong filamentation effects associated with large core vorticity. Wang (2008) extended the work of R06 by applying the filamentation time diagnostic to a fully compressible, three-dimensional, nonhydrostatic model simulation and thereby clarifying the relative roles of filamentation processes and subsidence in the formation of a moat. Recently, Kuo et al. (2009) have shown that the rapid filamentation process tends to make an important contribution to the organization of the moat in strong typhoons, especially in cases where the maximum winds are greater than $130 \mathrm{kts}$.

The purpose of the present paper is to generalize the filamentation time diagnostic of R06 from two-dimensional, nondivergent, vorticity dynamics in Cartesian coordinates to threedimensional, divergent, potential vorticity dynamics in spherical/isentropic coordinates, and then to apply this diagnostic tool to observed synoptic scale flows involving trough thinning and cutoff low formation. The mathematical analysis is given in section 2 , and the examples of trough thinning in both the midlatitudes and the tropics are presented in section 3 . The effects of the earth's curvature, divergence, and shearing/stretching deformations are discussed in section 4 , and a summary and concluding remarks are given in section 5 . 


\section{Theoretical concepts}

Inviscid, adiabatic, quasi-static flow in the atmosphere obeys the material conservation relation

$$
\frac{D P}{D t}=0
$$

where

$$
P=\left(-\frac{1}{g} \frac{\partial p}{\partial \theta}\right)^{-1}\left(2 \Omega \sin \phi+\frac{\partial v}{a \cos \phi \partial \lambda}-\frac{\partial(u \cos \phi)}{a \cos \phi \partial \phi}\right)
$$

is the potential vorticity and

$$
\frac{D}{D t}=\frac{\partial}{\partial t}+u \frac{\partial}{a \cos \phi \partial \lambda}+v \frac{\partial}{a \partial \phi}
$$

is the material derivative, with $g$ denoting the acceleration of gravity, $p$ the pressure, $\theta$ the potential temperature, $\Omega$ the earth's rotation rate, $a$ the earth's radius, $\lambda$ the longitude, $\phi$ the latitude, $u$ and $v$ the zonal and meridional components of the wind, and with the partial derivatives with respect to $(\lambda, \phi, t)$ taken on the isentropic surface. To understand the evolution of the PV gradient, we first differentiate (1) with respect to $\lambda$ and $\phi$ to obtain

$$
\frac{D}{D t}\left(\begin{array}{c}
\frac{\partial P}{a \cos \phi \partial \lambda} \\
\frac{\partial P}{a \partial \phi}
\end{array}\right)+\left(\begin{array}{cc}
\frac{\partial u}{a \cos \phi \partial \lambda}-\frac{v \tan \phi}{a} & \frac{\partial v}{a \cos \phi \partial \lambda} \\
\frac{\partial u}{a \partial \phi}+\frac{u \tan \phi}{a} & \frac{\partial v}{a \partial \phi}
\end{array}\right)\left(\begin{array}{c}
\frac{\partial P}{a \cos \phi \partial \lambda} \\
\frac{\partial P}{a \partial \phi}
\end{array}\right)=0
$$

We now define the isentropic divergence on the sphere as

$$
\delta=\frac{\partial u}{a \cos \phi \partial \lambda}+\frac{\partial(v \cos \phi)}{a \cos \phi \partial \phi}
$$

the isentropic relative vorticity as

$$
\zeta=\frac{\partial v}{a \cos \phi \partial \lambda}-\frac{\partial(u \cos \phi)}{a \cos \phi \partial \phi}
$$

and the isentropic stretching and shearing deformation as

$$
S_{n}=\frac{\partial u}{a \cos \phi \partial \lambda}-\cos \phi \frac{\partial}{a \partial \phi}\left(\frac{v}{\cos \phi}\right)
$$


and

$$
S_{s}=\frac{\partial v}{a \cos \phi \partial \lambda}+\cos \phi \frac{\partial}{a \partial \phi}\left(\frac{u}{\cos \phi}\right)
$$

respectively (cf. Eq. (3.2.3) of Bluestein 1992 and Eq. (2) of Kunz et al. 2009). Then, the tensor appearing in (4) can be written in the form

$$
\mathcal{V}=\left(\begin{array}{cc}
\frac{1}{2}\left(\delta+S_{n}\right) & \frac{1}{2}\left(S_{s}+\zeta-\frac{2 u \tan \phi}{a}\right) \\
\frac{1}{2}\left(S_{s}-\zeta+\frac{2 u \tan \phi}{a}\right) & \frac{1}{2}\left(\delta-S_{n}\right)
\end{array}\right) .
$$

The eigenvalues of $\mathcal{V}$ are

$$
\mu=\frac{1}{2}\left\{\delta \pm\left[S_{n}^{2}+S_{s}^{2}-\left(\zeta-\frac{2 u \tan \phi}{a}\right)^{2}\right]^{1 / 2}\right\}
$$

When these two eigenvalues are almost constant along a trajectory, the solutions of (4) are approximately linear combinations of

$$
\begin{aligned}
\exp \left\{\frac{1}{2}\left[\delta \pm i\left(\left(\zeta-\frac{2 u \tan \phi}{a}\right)^{2}-S_{n}^{2}-S_{s}^{2}\right)^{1 / 2}\right] t\right\} \\
\quad \text { if }\left(\zeta-\frac{2 u \tan \phi}{a}\right)^{2}>S_{n}^{2}+S_{s}^{2} \text { (rotation dominated), }
\end{aligned}
$$

or linear combinations of

$$
\begin{array}{r}
\exp \left\{\frac{1}{2}\left[\delta \pm\left(S_{n}^{2}+S_{s}^{2}-\left(\zeta-\frac{2 u \tan \phi}{a}\right)^{2}\right)^{1 / 2}\right] t\right\} \\
\quad \text { if }\left(\zeta-\frac{2 u \tan \phi}{a}\right)^{2}<S_{n}^{2}+S_{s}^{2} \text { (strain dominated) } .
\end{array}
$$

For the strain dominated case, we can define the filamentation time as the e-folding time of the exponential solution (12), i.e.,

$$
\tau_{\text {fil }}=2\left\{\delta+\left[S_{n}^{2}+S_{s}^{2}-\left(\zeta-\frac{2 u \tan \phi}{a}\right)^{2}\right]^{1 / 2}\right\}^{-1} \text { for } S_{n}^{2}+S_{s}^{2}>\left(\zeta-\frac{2 u \tan \phi}{a}\right)^{2} .
$$

R06 used the term "filamentation time" for $\tau_{\text {fil }}$; for historical reasons, and to avoid confusion, we shall use the term "Okubo-Weiss filamentation time." Strain dominated regions, as defined by (13), can also be referred to as "filamentation zones." 
For the examples presented in section 3, the surface analyzed is an isentropic surface near the tropopause. The re-analysis data used here are from the European Center for MediumRange Weather Forecasts (ECMWF). The wind field on the isentropic surface is obtained by linear interpolation from adjacent isobaric surfaces, which are scanned from top to bottom because there may be an inversion region near the earth's surface.

It should be pointed out that Hua and Klein (1998) have derived a more accurate filamentation time by relaxing the assumption that, following a fluid parcel, the velocity gradients are changing slowly compared to the vorticity gradient. R06 and Rozoff (2007) have compared these two criteria for hurricane-like flows. The results show that the difference is not dramatic. Because the Hua-Klein filamentation time includes the effect of the Lagrangian acceleration tensor, it yields somewhat larger rapid filamentation zones and smaller values of $\tau_{\text {fil }}$. The calculation of the Hua-Klein filamentation time involves the material time derivative of the deformation and the vorticity. Because the time resolution of the ECMWF re-analysis data is 6 hours, which is too coarse to accurately calculate the material time derivative, we concentrate here on the Okubo-Weiss filamentation time as our diagnostic parameter.

\section{Trough thinning in the midlatitudes and the tropics}

The first example is a strong baroclinic event near the European continent. This event was well studied in T93 and in James (1994, hereafter J94, his Figure 9.17). The ECMWF re-analysis data used in this example had a grid spacing of $2.5^{\circ}$ in both directions. Polar projection plots on the $310 \mathrm{~K}$ isentropic surface were made for easy comparison between our filamentation time diagnosis and those of T93 and J94. Of particular interest are the filamentation regions indicated by "A", "B", "C", and "D" in Fig. 1. Region A is at higher latitudes and region B is in an elongated trough at lower latitudes. There is cross-PV-contour flow behind the trough and more parallel-PV-contour flow ahead in both regions A and B. 
These are the trough thinning regions according to T93 and J94. The filamentation time (shading), which is calculated from (13), is between 1 and 2 hours (Fig. 1b). In the unshaded areas, the flow is either vorticity dominated or has a filamentation time that is negative or longer than 4 hours.

Even with a filamentation time of $1-2$ hours and a subsequent increase of the PV gradient in the high latitude region A, the trough does not cutoff. ${ }^{1}$ On the other hand, region $\mathrm{B}$ is associated with a equatorward migrating trough (Fig. 1a and Fig. 1b). At the narrowest part of the trough, near $3 \mathrm{~W}, 45 \mathrm{~N}$ in Fig. $1 \mathrm{~b}$, the filamentation time is $1-2$ hours. Twelve hours later (Fig. 1c), the trough has cutoff, with the cutoff low eventually drifting southward away from the original trough (Fig. 1d). It appears that the cutoff low separates from the main system in the region of rapid filamentation in the NE-SW elongated equatorward migrating trough. West of the thinning trough, a trough centered at $50 \mathrm{~W}, 47 \mathrm{~N}$ has cross-PV-contour flow on its east side and more parallel-PV-contour flow on its south side. This is the broadening trough situation discussed by T93 and J94. For this broadening trough, filamentation zones are found outside the circumference of the 1 PVU contour (region C).

The broadening trough that is connected to the high-PV reservoir to the northwest at $45 \mathrm{~W}, 55 \mathrm{~N}$ (region D) in Fig. 1 is another example of a trough that does not cutoff in spite of short filamentation times. The narrowest width of 2 PVU contours in the D region near $50 \mathrm{~W}, 52 \mathrm{~N}$, thins from $600 \mathrm{~km}$ to $400 \mathrm{~km}$ in a 12 hour period (e.g., Fig. 1b and Fig. 1c) and then increases to $800 \mathrm{~km}$ in the following 12 hour period (e.g., Fig. 1c and Fig. 1d). The trough does not cutoff, even though the diagnosed filamentation times are short. This complication may arise from the fact that there is strong positive vorticity advection at 50W, $52 \mathrm{~N}$ in Fig. 1c, which may change the pattern of the PV gradient in the filamentation zone.

\footnotetext{
${ }^{1}$ Here we define a cutoff low as a displacement from the main system by $1 \mathrm{PVU}$ on the $310 \mathrm{~K}$ and 330 $\mathrm{K}$ isentropic surfaces in midlatitudes, and by $2 \mathrm{PVU}$ on the $360 \mathrm{~K}$ isentropic surface in tropics, where PVU stands for a "PV unit" of $10^{-6} \mathrm{~m}^{2} \mathrm{~s}^{-1} \mathrm{~K} \mathrm{~kg}^{-1}$.
} 
In addition, the filamentation zone is on the cyclonic side of the jet stream and is subject to trough broadening according to T93. Region A is also on the cyclonic side of the jet stream. Thus, it appears that regions on the cyclonic shear side of the jet stream may not cutoff even with a short filamentation time.

The filamentation zones in this example occur in the trough cutoff region of the thinning trough (region B) and outside the circumference of a broadening trough (region $\mathrm{C}$ ). The filamentation time diagnosis agrees with the thinning and broadening dynamics of T93 and J94. These analyses demonstrate that it is useful to combine the cross-PV-contour flow analysis on isentropic surfaces (T93) with the filamentation time analysis. Our results suggest that cutoffs occur when there are short filamentation times on the anticyclonic side of the jet stream in equatorward migrating troughs.

As a second example, we present a midlatitude event with moderate baroclinicity near Taiwan during the Mei-Yu period of early May 1998. This event was characterized by both trough thinning and cutoff low formation. The ECMWF re-analysis data used in this example had a grid spacing of $0.5^{\circ}$ in both directions. In Fig. 2a, the trough is located between $25 \mathrm{~N}$ and $50 \mathrm{~N}$. On the $330 \mathrm{~K}$ isentropic surface, high cross-contour winds occur in the northwest sector and weaker cross-contour winds occur to the south. This general pattern has been discussed in T93 (see their schematic Fig. 13), and the trough is predicted to thin with time. At the narrowest part of the trough, near $117 \mathrm{E}, 38 \mathrm{~N}$, the filamentation time (shading) is between 3 and 6 hours. There is a strong tendency for the deformation field to stretch the trough. Twelve hours later (Fig. 2b) the trough has stretched, but still has large cross-PV-contour winds on its northwest side. Twelve hours later, Fig. 2c reveals a thin PV filament. At this stage, the wind vectors still cross PV contours near the upper part of the trough, but are parallel to the trough on the southeast side, which means the trough will continue to thin. The similar 3 to 6 hour filamentation times in Fig. 2a and Fig. 2c indicate that high cross-contour winds in the north with weaker cross-contour winds to the 
south (Fig. 2a) can lead to a thinning of the trough just as much as weak cross-contour winds in the north and along-contour flow to the south (Fig. 2c). The shortest filamentation times are located in the region where spatial variation of cross PV contour flow at both sides of the trough is largest. Figure $2 \mathrm{~d}$ which is 36 hours from the beginning, shows that a cutoff low has separated from the trough, and regions with short filamentation times surround the cutoff low and the trough.

The third example presented here is a TUTT event over the Atlantic Ocean during June 2005. TUTTs, also called midoceanic troughs, are elongated troughs over the tropical oceans. Such troughs often interact strongly with tropical cyclones, as discussed in the comprehensive study of Hanley et al. (2001). In the example presented here, a low latitude cutoff low (a so-called "TUTT cell") also formed. The ECMWF re-analysis data used in this example had a grid spacing of $1.125^{\circ}$ in both directions. Fig. 3 depicts the evolution of this event on the $360 \mathrm{~K}$ isentropic surface. This isentropic surface is higher than the midlatitude case since the altitude of the tropopause is higher in the tropics.

At 12 UTC on 2 June 2005 (Fig. 3a), the TUTT was located between 40W and 77W, and between $8 \mathrm{~N}$ and $30 \mathrm{~N}$, with small filamentation times $\left(\tau_{\text {fil }} \approx 12-24\right.$ hours $)$ located near $58 \mathrm{~W}$, $17 \mathrm{~N}$, just north of the TUTT cell. This filamentation zone suggests that the trough will thin with time. Twelve hours later (Fig. 3b), the TUTT has further elongated in the NE-SW direction. The shortest filamentation times ( $\tau_{\text {fil }}$ less than 12 hours) are located near 57W, $19 \mathrm{~N}$, just at the region separating the TUTT and the TUTT cell. Note that the narrowest width of the 3 PVU contour in the filamentation zone changes from approximately $400 \mathrm{~km}$ to $200 \mathrm{~km}$ in 12 hours (e.g., Fig. 3a and 3b). The PV gradient growth is about a factor of 2 in 12 hours. Although a PV gradient growth of a factor of 2 is smaller than the $e^{1}$ factor, the fine structure of PV may be affected by PV debris from diabatic processes and by data resolution errors. One day later (Fig. 3c), in the gap region near $62 \mathrm{~W}$, $19 \mathrm{~N}$, the filamentation time has decreased to less than 12 hours, which prevents the TUTT cell from 
merging again with the TUTT. In fact, the 3 PVU contour is cutoff at this time. Still later (Fig. 3d), the TUTT and TUTT cell are nearly separated, and the filamentation time is still less than 12 hours. Eventually (Fig. 3e) the TUTT and the TUTT cell become separate with a filamentation time less than 18 hours surrounding the TUTT cell.

Note that the cutoff region in Fig. 2c is associated with a very sharp filamentation zone centered on the highest PV gradient area, while that in Fig. 3d is embedded in a broader

filamentation zone. Yet both troughs cutoff in 12 hours. A careful examination of Fig. 3d indicates that the rapid filamentation zone $\left(\tau_{\text {fil }}<12\right.$ hours $)$ is also centered on the highest PV gradient area. The significance of the surrounding filamentation zone $\left(\tau_{\text {fil }}>12\right.$ hours) in Fig. 3d appears to play a secondary role in the cutoff process.

The above examples indicate that the filamentation time in the tropical event is generally longer than in the midlatitude events, which have stronger baroclinicity. This may be caused by the generally weaker winds (also weaker deformation fields) in the tropics, which make the filamentation time longer. It is also interesting to note that the TUTT cell in this event forms an approximately elliptical vortex. An elliptical vortex will rotate counterclockwise with time (Kuo et al. 1999), an effect that can be clearly seen in Fig. 3d - 3e.

\section{Discussion}

For synoptic scale weather phenomenon, scale analysis indicates that the vorticity and deformation fields are usually an order of magnitude larger than the divergence, an order of magnitude larger than the curvature terms in midlatitudes, and two orders of magnitude larger than the curvature terms in the tropics. It is interesting to calculate the importance of the curvature terms and the divergence term on the filamentation time. If we neglect all 
the curvature terms in (10), we obtain

$$
\begin{aligned}
\mu & =\frac{1}{2}\left\{\left(\frac{\partial u}{a \cos \phi \partial \lambda}+\frac{\partial v}{a \partial \phi}\right) \pm\left[\left(\frac{\partial u}{a \cos \phi \partial \lambda}-\frac{\partial v}{a \partial \phi}\right)^{2}\right.\right. \\
& \left.\left.+\left(\frac{\partial v}{a \cos \phi \partial \lambda}+\frac{\partial u}{a \partial \phi}\right)^{2}-\left(\frac{\partial v}{a \cos \phi \partial \lambda}-\frac{\partial u}{a \partial \phi}\right)^{2}\right]^{1 / 2}\right\}
\end{aligned}
$$

The eigenvalues computed from (14) are almost identical to those computed from (10) for midlatitude troughs and TUTT events, so we conclude that the curvature terms are not important in the filamentation time calculation. On the other hand, if we do not consider the divergence term but keep all the curvature terms, (10) reduces to

$$
\begin{aligned}
\mu & = \pm \frac{1}{2}\left\{\left[\left(\frac{\partial u}{a \cos \phi \partial \lambda}-\frac{\partial v}{a \partial \phi}-\frac{v \tan \phi}{a}\right)^{2}\right.\right. \\
& \left.\left.+\left(\frac{\partial v}{a \cos \phi \partial \lambda}+\frac{\partial u}{a \partial \phi}+\frac{u \tan \phi}{a}\right)^{2}-\left(\frac{\partial v}{a \cos \phi \partial \lambda}-\frac{\partial u}{a \partial \phi}-\frac{u \tan \phi}{a}\right)^{2}\right]^{1 / 2}\right\} .
\end{aligned}
$$

Filamentation times based on (15) are shown in Fig. 4 for both the midlatitude baroclinic trough event and the TUTT event. Comparing Fig. 4a with Fig. 2b, we find that, without the isentropic divergence term, the filamentation times become shorter (longer) due to the convergent (divergent) effect in the cutoff region, resulting in some unrealistic rapid filamentation zones. Comparing Fig. 4b with Fig. 3c, we also find the isentropic divergence term changes both the value and distribution of the filamentation time, resulting in a slightly larger filamentation zone in the TUTT cell cutoff region. The differences between non-divergent and divergent cases are further calculated in the cutoff regions with PV larger than 1 PVU (2 PVU) in midlatitude (tropics). We have found that the difference in our first case (region B) is $21 \%$, second case $14 \%$, and the third case $12 \%$. There are approximately $10 \%$ to $20 \%$ differences in the cutoff regions without the divergence term in these cases. As the result, neglect of curvature terms for simplicity is acceptable if the event is not too close to the pole, but retaining the isentropic divergence term is helpful for increased accuracy.

In the second midlatitude event, the cutoff low only moves $8^{\circ}$ in the zonal direction (from $110 \mathrm{E}$ to $118 \mathrm{E}$ ) and slightly southward near $30 \mathrm{~N}$ during a 36 hour period. On the 
other hand, the center of the trough moves from $122 \mathrm{E}, 42 \mathrm{~N}$ to $138 \mathrm{E}, 40 \mathrm{~N}$, i.e., the trough moves $16^{\circ}$ in the zonal direction near $40 \mathrm{~N}$. The trough is thus in a NE-SW orientation. The local orientation of the trough distinguishes the two types of deformation, with the EastWest or North-South orientated trough being dominated by shearing deformation, and the NE-SW orientated trough being dominated by the stretching deformation. This means that stretching deformation plays a dominant role in the NE-SW oriented trough thinning process. Midlatitude troughs are often NE-SW oriented and thus dominated by stretching deformation (e.g., Fig. 5a). This may be the reason Kunz et al. (2009) used stretching deformation based on spherical coordinates to diagnose wave breaking events. On the other hand, the filamentation zone in the TUTT case (e.g., Fig. 5b and 5c) shows both the stretchingdominant and shearing-dominant regions depending on the local orientation of the trough. This is in contrast to the shearing deformation dominating the circumference of midlatitude broadening lows and in tropical cyclones immediately outside the eyewall. Since coordinate rotations transfer one deformation component into the other (Bluestein 1992), it may be argued that it is of no interest to distinguish between the two types of deformations. Values of $S_{n}$ and $S_{s}$ are dependent on the orientation of the trough, but the value of $\left(S_{n}^{2}+S_{s}^{2}\right)^{1 / 2}$ is not. Thus, an advantage of the filamentation time analysis is that it combines both deformations into a single diagnostic quantity.

\section{$5 \quad$ Summary and concluding remarks}

In this study the filamentation time in spherical coordinates on an isentropic surface has been derived and used as a diagnostic tool to analyze trough thinning processes for both midlatitude and tropical cases. Of the three cases with strong, moderate, and weak baroclinicities, the filamentation times at the cutoff region are $1-2$ hours, $3-6$ hours, and $6-$ 12 hours respectively. The troughs did cut off in $6-12$ hours (12 - 24 hours) for the strong and moderate (weak) baroclinicity cases. It has been found that the trough thinning and the 
cutoff low formation processes can be quantified by the filamentation time diagnostic. Our results suggest that cutoff low formation occurs with short filamentation times in equatorward migrating troughs on the anticyclonic side of the jet stream. Furthermore, our analysis indicates that the filamentation time is generally longer in the tropics than in midlatitudes, which means the cutoff low formation process is slower in the tropics than in midlatitudes due to the weaker deformation wind fields in the tropics.

Because the curvature terms are only significant at high latitudes, they can be ignored for simplicity, whereas the isentropic divergence term should be retained for increased accuracy. We have also used different resolution data to calculate the filamentation time in the second midlatitude case. Using $2.5^{\circ} \times 2.5^{\circ}$ data, the filamentation time and rapid filamentation zone do not change much, which means that this resolution is in general adequate for qualitative diagnosis of the filamentation time. An improved quantitative comparison can be made with higher temporal resolution data from a numerical simulation. The higher temporal resolution data could also be used to check the validity of the "slowly varying velocity gradients" assumption.

In addition to the PV dynamics of T93, the filamentation time diagnostic contains the additional effects of divergence, stretching and shearing deformations. The results of this paper indicate that the filamentation time diagnostic on isentropic potential vorticity maps is a useful tool for the analysis of trough thinning processes in both the midlatitudes and the tropics. A combination of cross-PV-contour flow analysis on isentropic surfaces with the filamentation time analysis gives a more complete description of the synoptic dynamics.

Acknowledgments. We are grateful to Mark I.-M. Wang, Paul Ciesielski, Yi-Ting Yang, and Brian McNoldy for many helpful discussions. We wish to thank two anonymous reviewers 
for many helpful comments. This research was supported by the National Science Council of Taiwan through Grants NSC-096-2917-I-002-129, NSC-097-2111-M-002-007, NSC-097-2628M-002-023, 97R0066-69, and by the US National Science Foundation under Grant ATM0837932 . 


\section{REFERENCES}

Appenzeller, C., H. C. Davies, and W. Norton, 1996: Fragmentation of stratospheric intrusion. J. Geophys. Res., 101, 1435-1456.

Bluestein, H., 1992: Synoptic-Dynamic Meteorology in Midlatitudes, Volume 1. Oxford University Press, 431 pp.

Ferreira, R. N., and W. H. Schubert, 1999: The role of tropical cyclones in the formation of tropical upper-tropospheric troughs. J. Atmos. Sci., 56, 2891-2907.

Guinn, T. A., and W. H. Schubert, 1993: Hurricane spiral bands. J. Atmos. Sci., 50, $3380-3403$.

Hanley, D., J. Molinari, and D. Keyser, 2001: A composite study of the interactions between tropical cyclones and upper-tropospheric troughs. Mon. Wea. Rev., 129, 2570-2584.

Hoskins, B. J., 1991: Towards a PV- $\theta$ view of the general circulation. Tellus, 43, 27-35.

Hua, B. L., and P. Klein, 1998: An exact criterion for the stirring properties of nearly two-dimensional turbulence. Physica D, 113, 98-110.

James, I. N., 1994: Introduction to circulating atmospheres. Cambridge University Press, 422 pp.

Kelley, W. E., and D. R. Mock, 1982: A diagnostic study of upper tropospheric cold lows over the western North Pacific. Mon. Wea. Rev., 110, 471-480.

Keyser, D., and M. A. Shapiro, 1986: A review of the structure and dynamics of upper-level frontal zones. Mon. Wea. Rev., 114, 452-499.

Koh, T. Y., and B. Legras, 2002: Hyperbolic lines and the stratospheric polar vortex. Chaos, 12(2), 382-394. 
Kunz, T., K. Fraedrich, and F. Lunkeit, 2009: Synoptic scale wave breaking and its potential to drive NAO-like circulation dipoles: A simplified GCM approach. Q. J. R. Meteorol. Soc., 135, 1-19.

Kuo, H.-C., R. T. Williams, and J.-H. Chen, 1999: A possible mechanism for the eye rotation of typhoon Herb. J. Atmos. Sci., 56, 1659-1673.

Kuo, H.-C., C.-P. Chang, Y.-T. Yang, and H.-J. Jiang, 2009: Western North Pacific typhoons with concentric eyewalls. Mon. Wea. Rev., 137, in press.

McWilliams, J. C., 1984: The emergence of isolated coherent vortices in turbulent flow. J. Fluid Mech., 146, 21-43.

Okubo, K., 1970: Horizontal dispersion of floatable particles in the vicinity of velocity singularities such as convergence. Deep-Sea Res., 17, 445-454.

Rozoff, C. M., W. H. Schubert, and B. D. McNoldy, 2006: Rapid filamentation zones in intense tropical cyclones. J. Atmos. Sci., 63, 325-340.

Rozoff, C. M. 2007: Aspects of moat formation in tropical cyclone eyewall replacement cycles. Colorado State University, Department of Atmospheric Science, PhD. Dissertation, $165 \mathrm{pp}$.

Thorncroft, C. D., B. J. Hoskins, and M. E. McIntyre, 1993: Two paradigms of baroclinicwave life-cycle behaviour. Q. J. R. Meteorol. Soc., 119, 17-55.

Wang, Y., 2008: Rapid filamentation zone in a numerically simulated tropical cyclone. J. Atmos. Sci., 65, 1158-1181.

Weiss, J., 1991: The dynamics of enstrophy transfer in two-dimensional hydrodynamics. Physica D, 48, 273-294. 


\section{Figure Captions}

Figure 1. Filamentation time in hours (shading), potential vorticity (black contours, with 1, 2, 4, 6, 8 PVU), where PVU stands for a "PV unit" of $10^{-6} \mathrm{~m}^{2} \mathrm{~s}^{-1} \mathrm{~K} \mathrm{~kg}^{-1}$, and wind vectors in $\mathrm{m} \mathrm{s}^{-1}$ on the $310 \mathrm{~K}$ isentropic surface for (a) 28 Jan $199000 \mathrm{UTC}$, (b) 28 Jan 1990 12 UTC, (c) 29 Jan 199000 UTC, (d) 29 Jan 199012 UTC. In the unshaded areas, the flow is either vorticity dominated or has a filamentation time that is negative or longer than 4 hours. The scale for the wind vectors $\left(50 \mathrm{~m} \mathrm{~s}^{-1}\right)$ is given at the bottom of the figure.

Figure 2. Filamentation time in hours (shading), potential vorticity (black contours, with a contour interval of 0.5 PVU starting at $0.5 \mathrm{PVU}$ ), and wind vectors in $\mathrm{m} \mathrm{s}^{-1}$ on the $330 \mathrm{~K}$ isentropic surface for (a) 4 May 199800 UTC, (b) 4 May 199812 UTC, (c) 5 May 1998 00 UTC, (d) 5 May 199812 UTC. In the unshaded areas, the flow is either vorticity dominated or has a filamentation time that is negative or longer than 9 hours. The bold solid line is the 1.5 PVU contour, which represents the dynamic tropopause. The scale for the wind vectors $\left(50 \mathrm{~m} \mathrm{~s}^{-1}\right)$ is given at the bottom of the figure.

Figure 3. Filamentation time in hours (shading), potential vorticity (black contours, with a contour interval of $1 \mathrm{PVU}$ starting at $2 \mathrm{PVU}$ ), and wind vectors in $\mathrm{m} \mathrm{s}^{-1}$ on the $360 \mathrm{~K}$ isentropic surface for (a) 2 June 200512 UTC, (b) 3 June 200500 UTC, (c) 3 June 2005 12 UTC, (d) 4 June 200500 UTC, (e) 4 June 200512 UTC. In the unshaded areas, the flow is either vorticity dominated or has a filamentation time that is negative or longer than 24 hours. The scale for the wind vectors $\left(50 \mathrm{~m} \mathrm{~s}^{-1}\right)$ is given at the bottom of the figure.

Figure 4. (a) The same as Fig. 2b but without the divergence term. (b) The same as Fig. 3c but without the divergence term. 
Figure 5. The potential vorticity (black contours, in PVU), and the deformation field (shading): (a) square of the stretching deformation in $10^{-8} \mathrm{~s}^{-2}$ for 4 May 199812 UTC; (b) square of the stretching deformation in $10^{-9} \mathrm{~s}^{-2}$ for 3 June 200512 UTC; (c) square of the shearing deformation in $10^{-9} \mathrm{~s}^{-2}$ for 3 June 200512 UTC. We only analyse the filamentation zone in the trough for the TUTT case. 


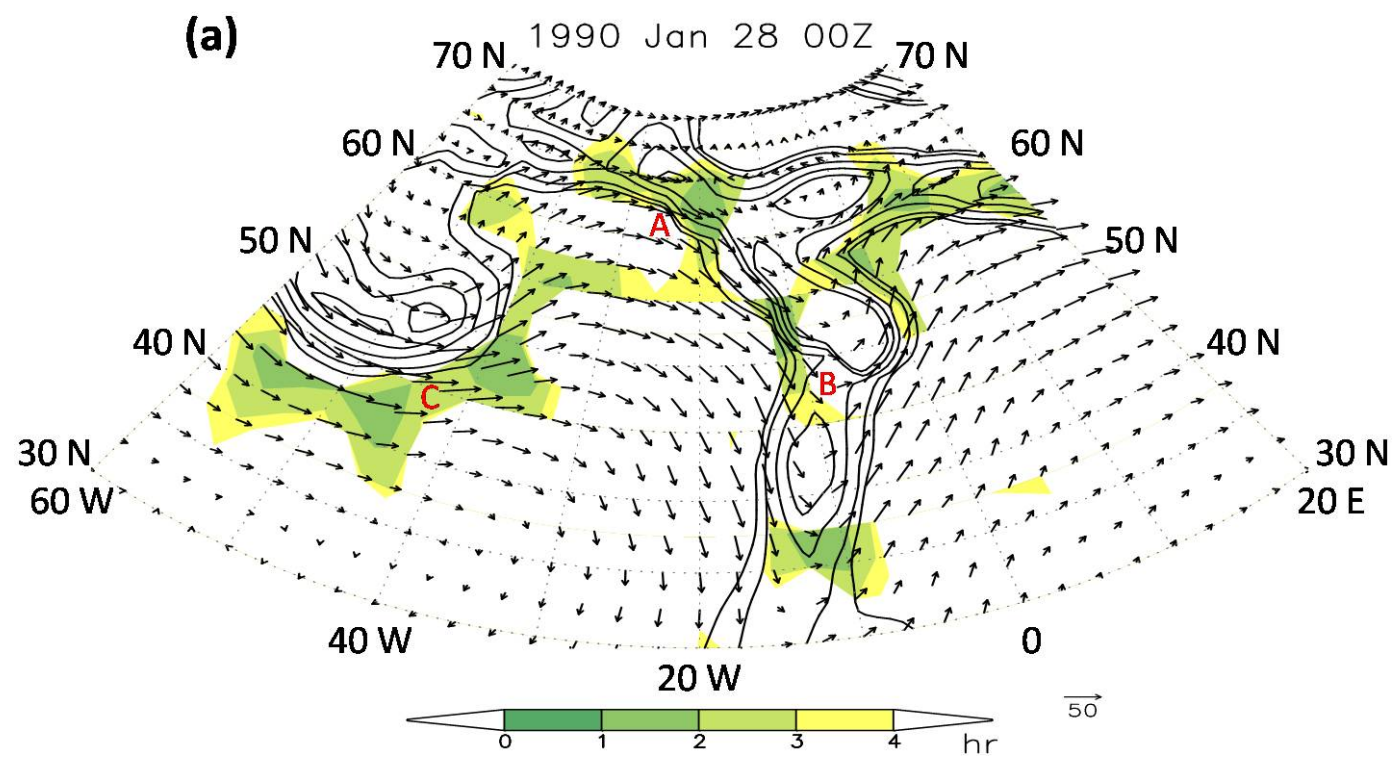

Figure 1: Filamentation time in hours (shading), potential vorticity (black contours, with 1, 2, 4, 6, 8 PVU), where PVU stands for a "PV unit" of $10^{-6} \mathrm{~m}^{2} \mathrm{~s}^{-1} \mathrm{~K} \mathrm{~kg}^{-1}$, and wind vectors in $\mathrm{m} \mathrm{s}^{-1}$ on the $310 \mathrm{~K}$ isentropic surface for (a) 28 Jan 199000 UTC, (b) 28 Jan 199012 UTC, (c) 29 Jan 199000 UTC, (d) 29 Jan 199012 UTC. In the unshaded areas, the flow is either vorticity dominated or has a filamentation time that is negative or longer than 4 hours. The scale for the wind vectors $\left(50 \mathrm{~m} \mathrm{~s}^{-1}\right)$ is given at the bottom of the figure. 


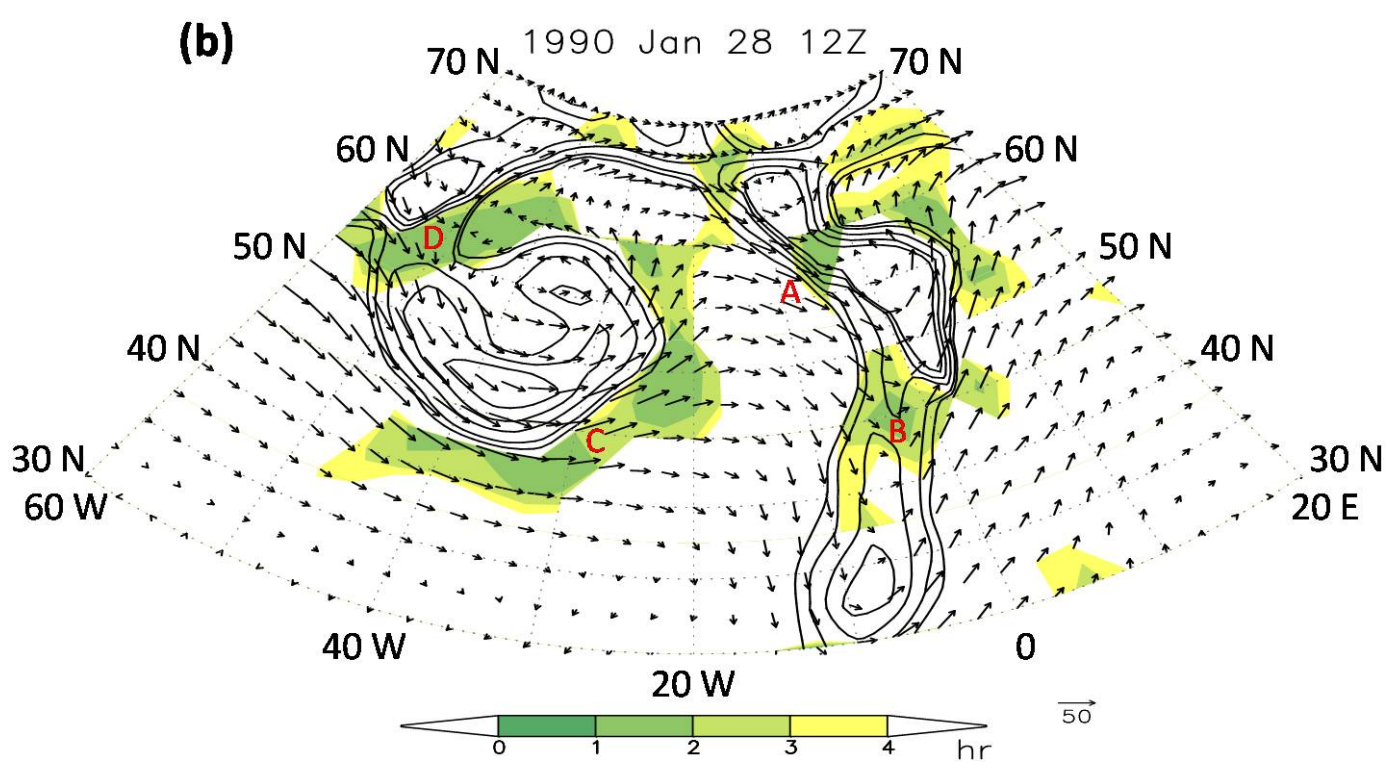

Figure 1: (Continued) 


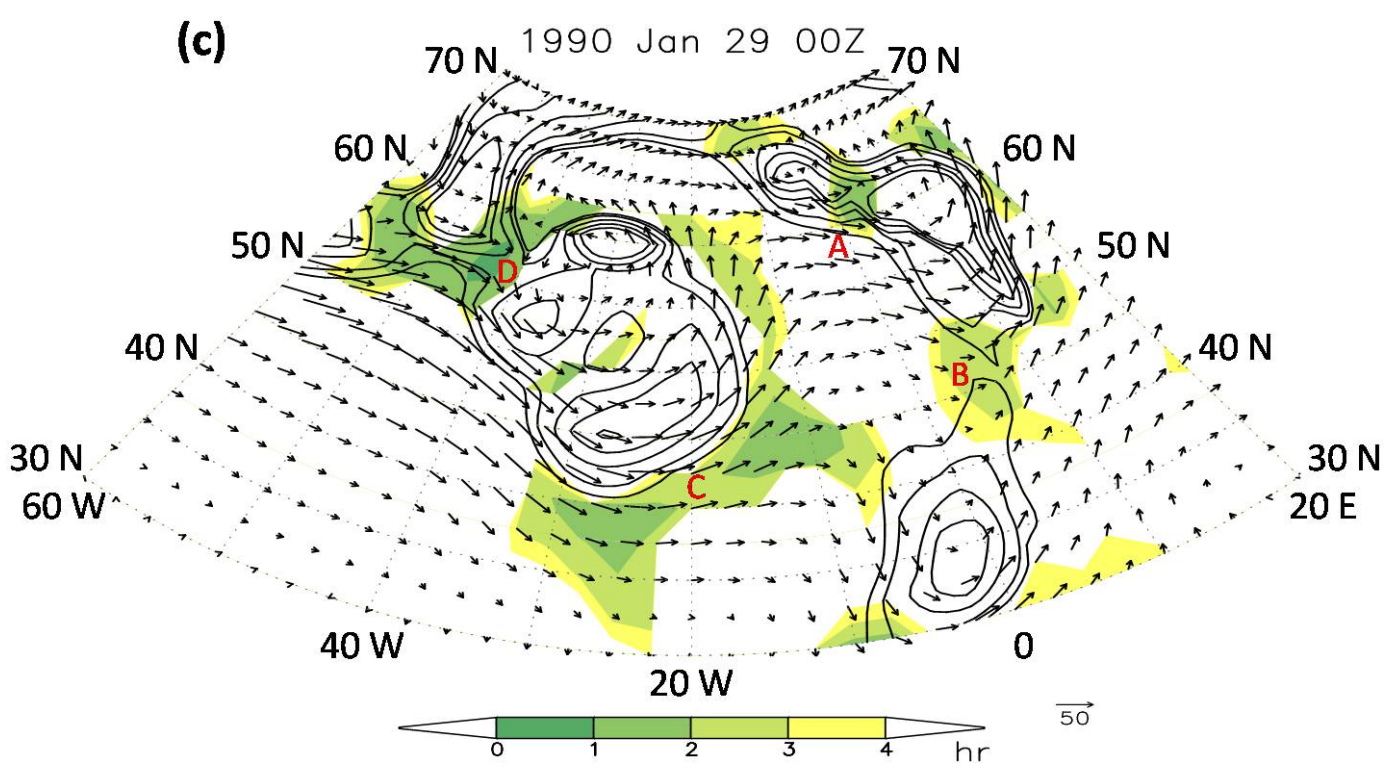

Figure 1: (Continued) 


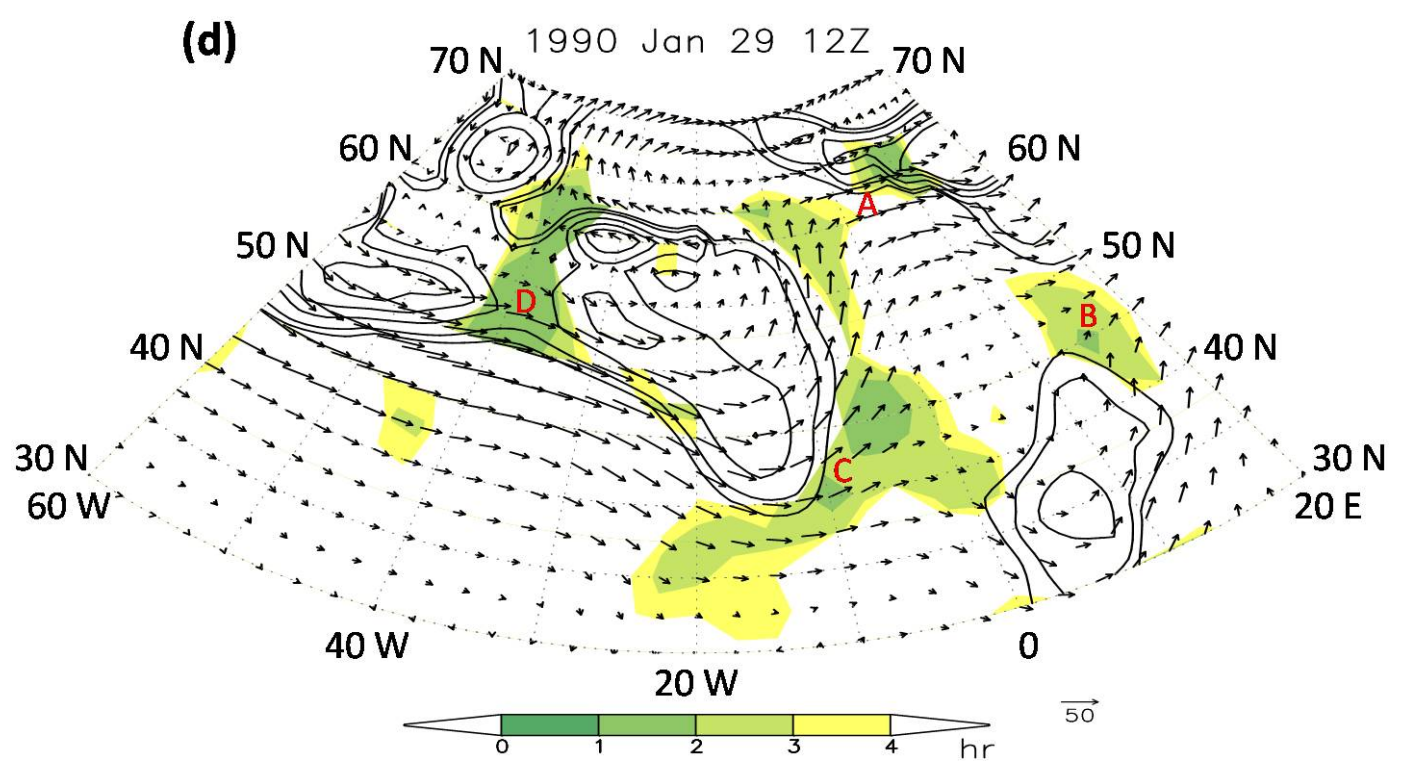

Figure 1: (Continued) 


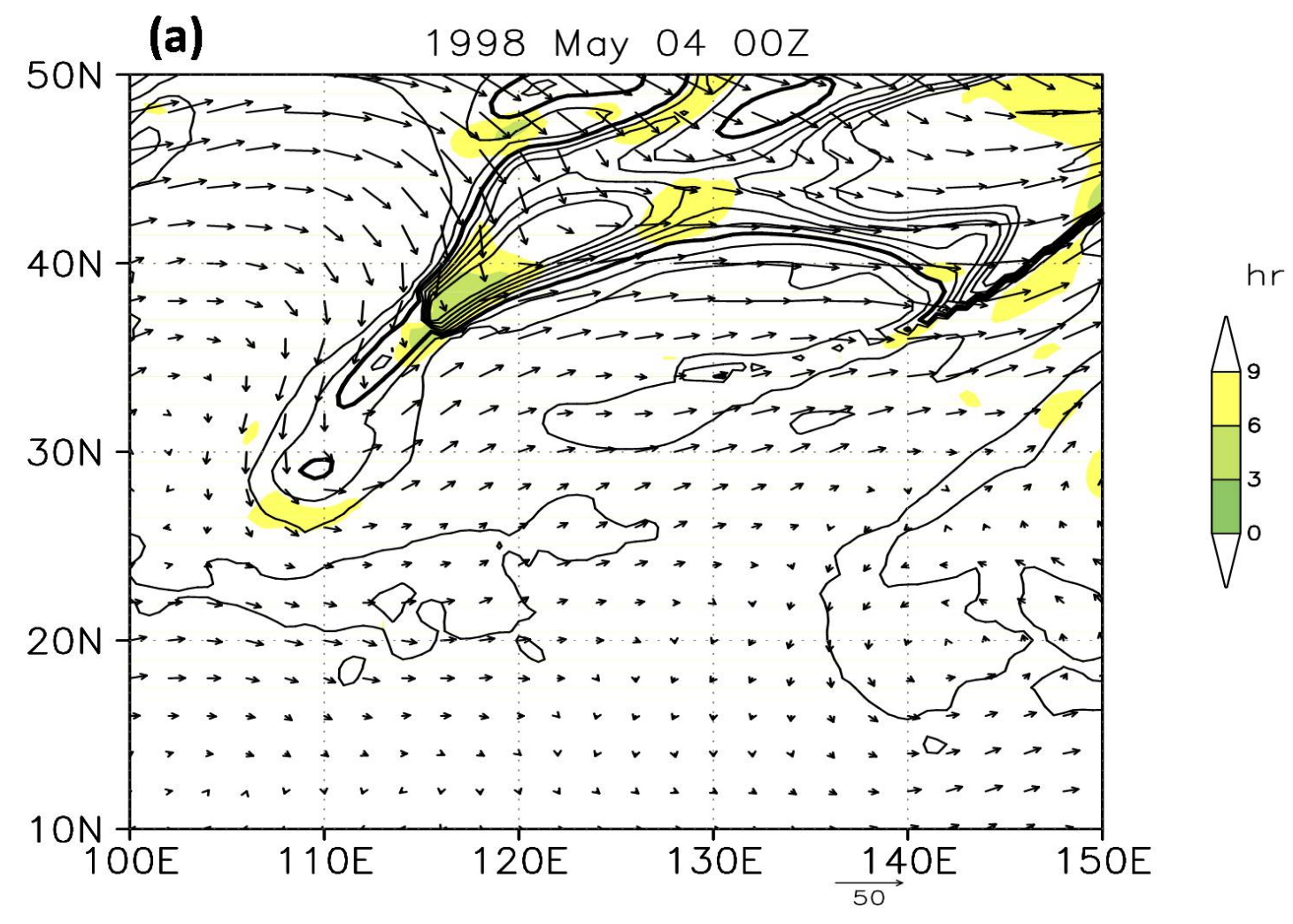

Figure 2: Filamentation time in hours (shading), potential vorticity (black contours, with a contour interval of $0.5 \mathrm{PVU}$ starting at $0.5 \mathrm{PVU}$ ), and wind vectors in $\mathrm{m} \mathrm{s}^{-1}$ on the $330 \mathrm{~K}$ isentropic surface for (a) 4 May 199800 UTC, (b) 4 May 199812 UTC, (c) 5 May 199800 UTC, (d) 5 May 199812 UTC. In the unshaded areas, the flow is either vorticity dominated or has a filamentation time that is negative or longer than 9 hours. The bold solid line is the 1.5 PVU contour, which represents the dynamic tropopause. The scale for the wind vectors $\left(50 \mathrm{~m} \mathrm{~s}^{-1}\right)$ is given at the bottom of the figure. 


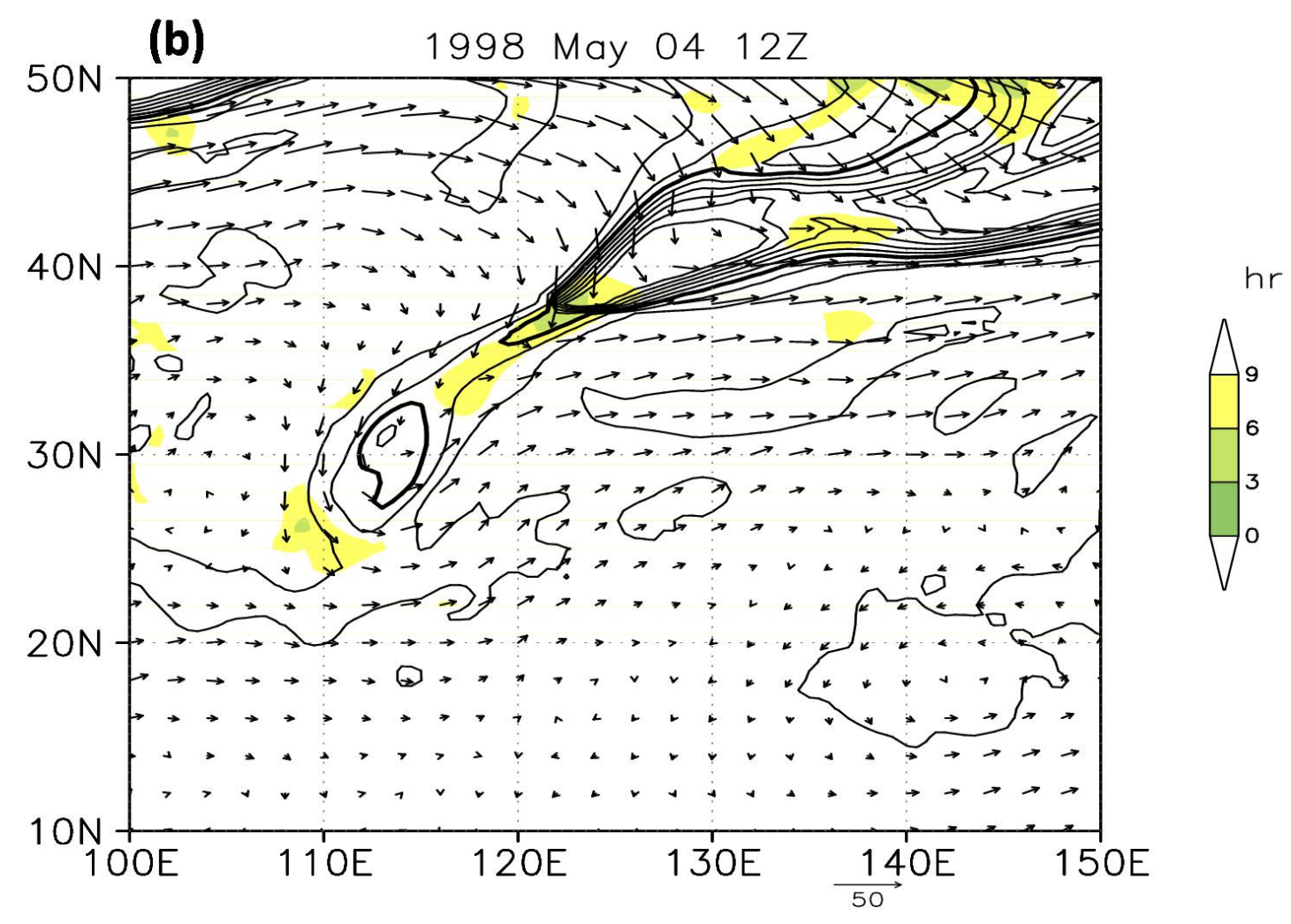

Figure 2: (Continued) 


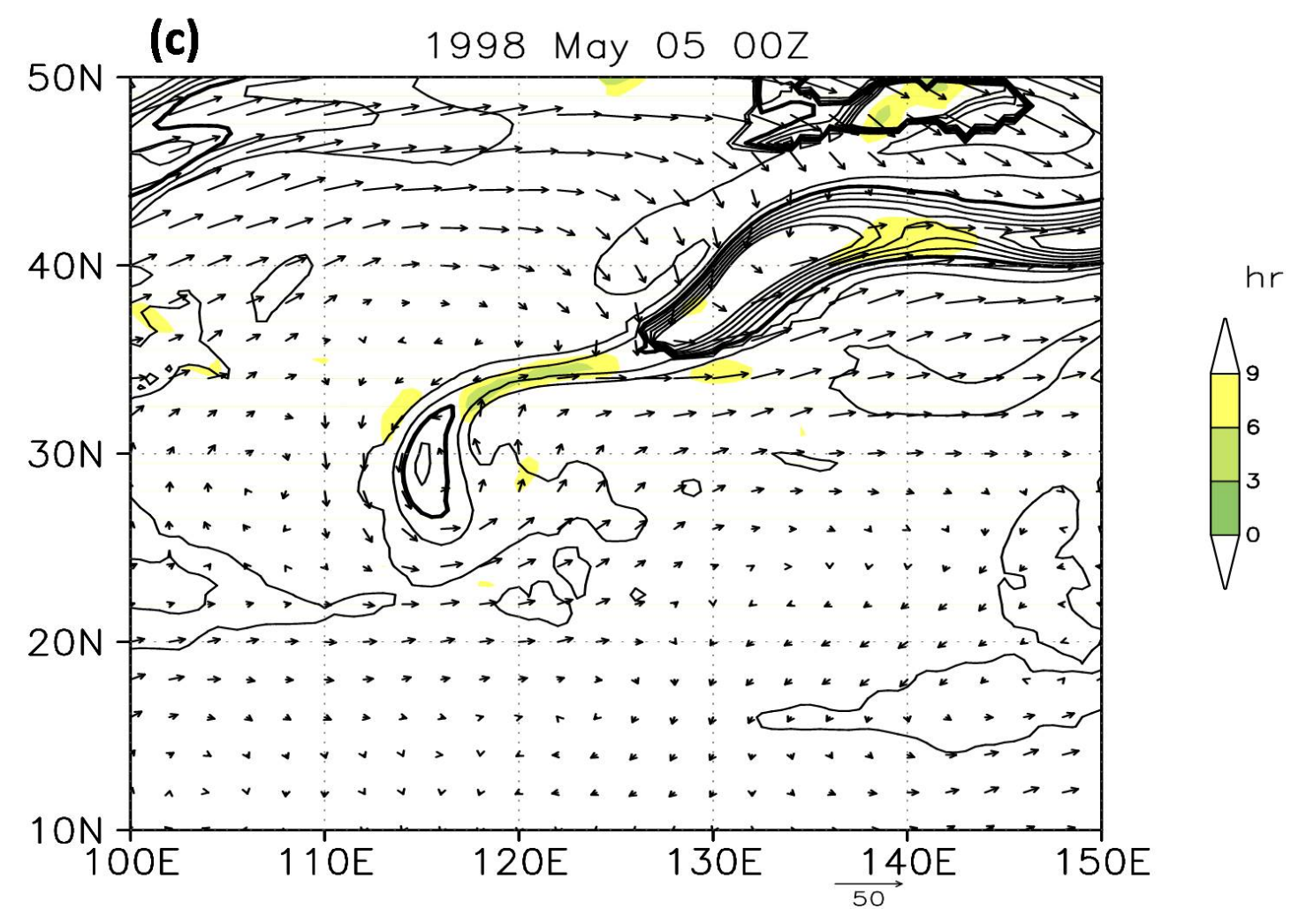

Figure 2: (Continued) 


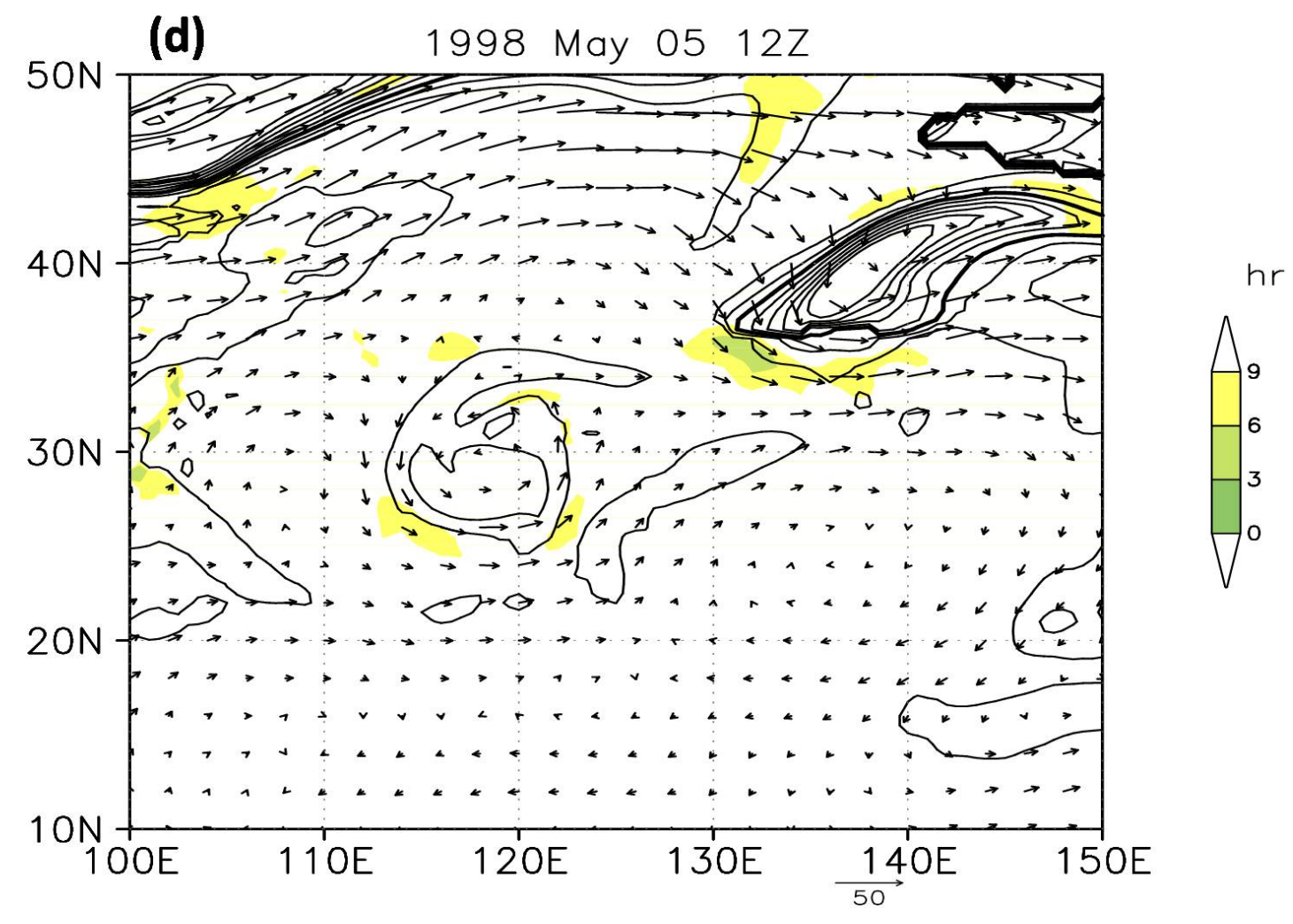

Figure 2: (Continued) 


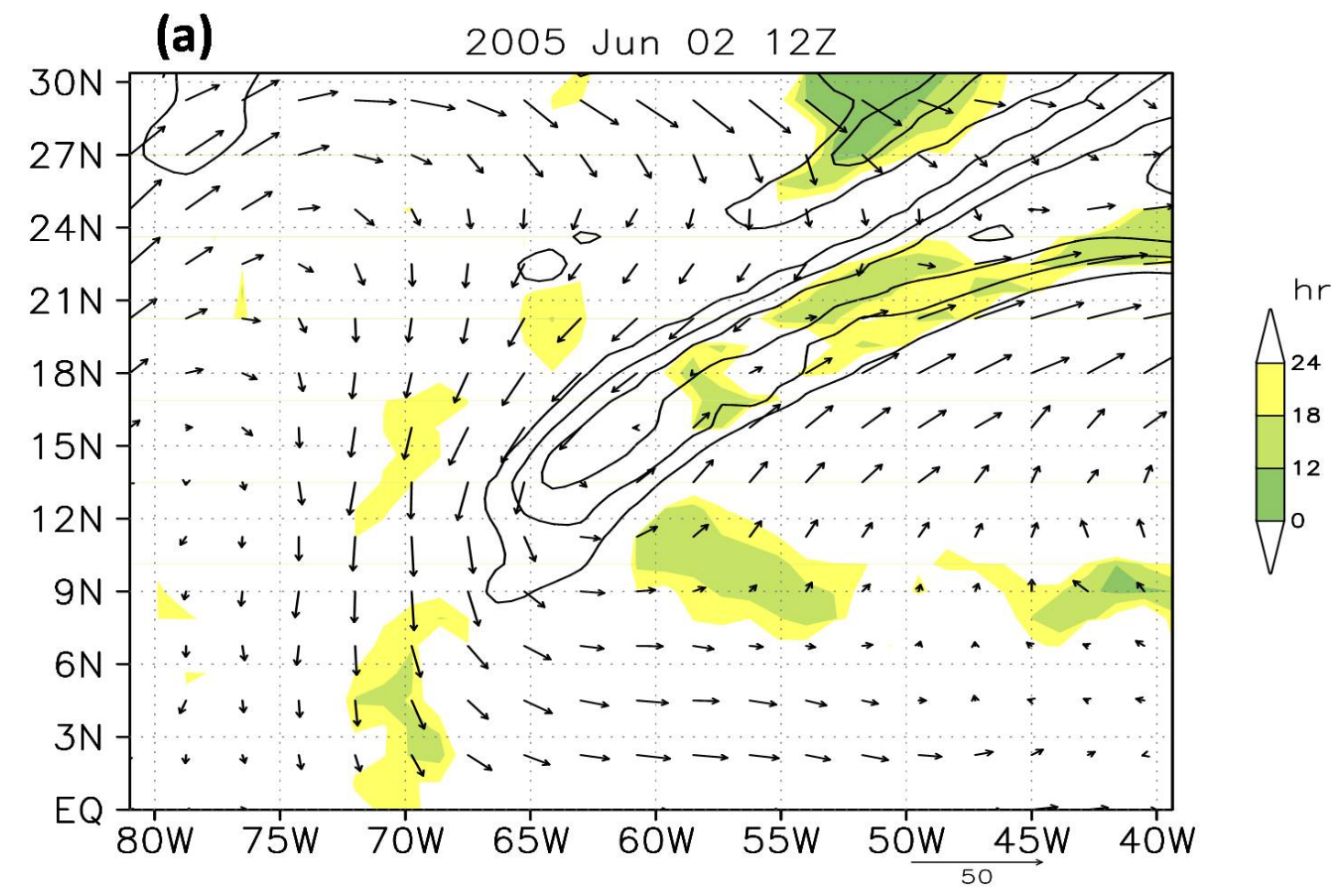

Figure 3: Filamentation time in hours (shading), potential vorticity (black contours, with a contour interval of $1 \mathrm{PVU}$ starting at $2 \mathrm{PVU}$ ), and wind vectors in $\mathrm{m} \mathrm{s}^{-1}$ on the $360 \mathrm{~K}$ isentropic surface for (a) 2 June 200512 UTC, (b) 3 June 200500 UTC, (c) 3 June 200512 UTC, (d) 4 June 200500 UTC, (e) 4 June 200512 UTC. In the unshaded areas, the flow is either vorticity dominated or has a filamentation time that is negative or longer than 24 hours. The scale for the wind vectors $\left(50 \mathrm{~m} \mathrm{~s}^{-1}\right)$ is given at the bottom of the figure. 


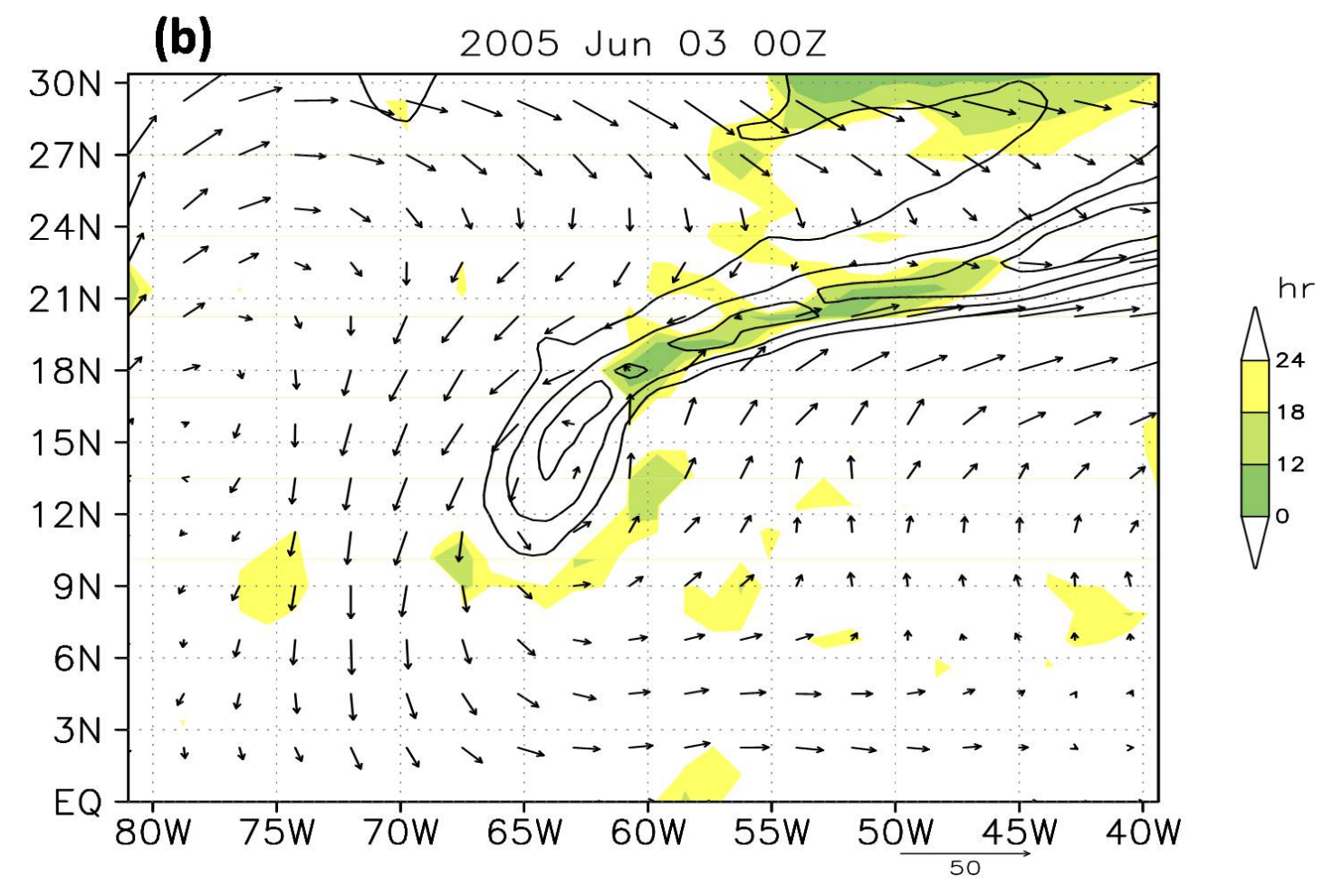

Figure 3: (Continued) 


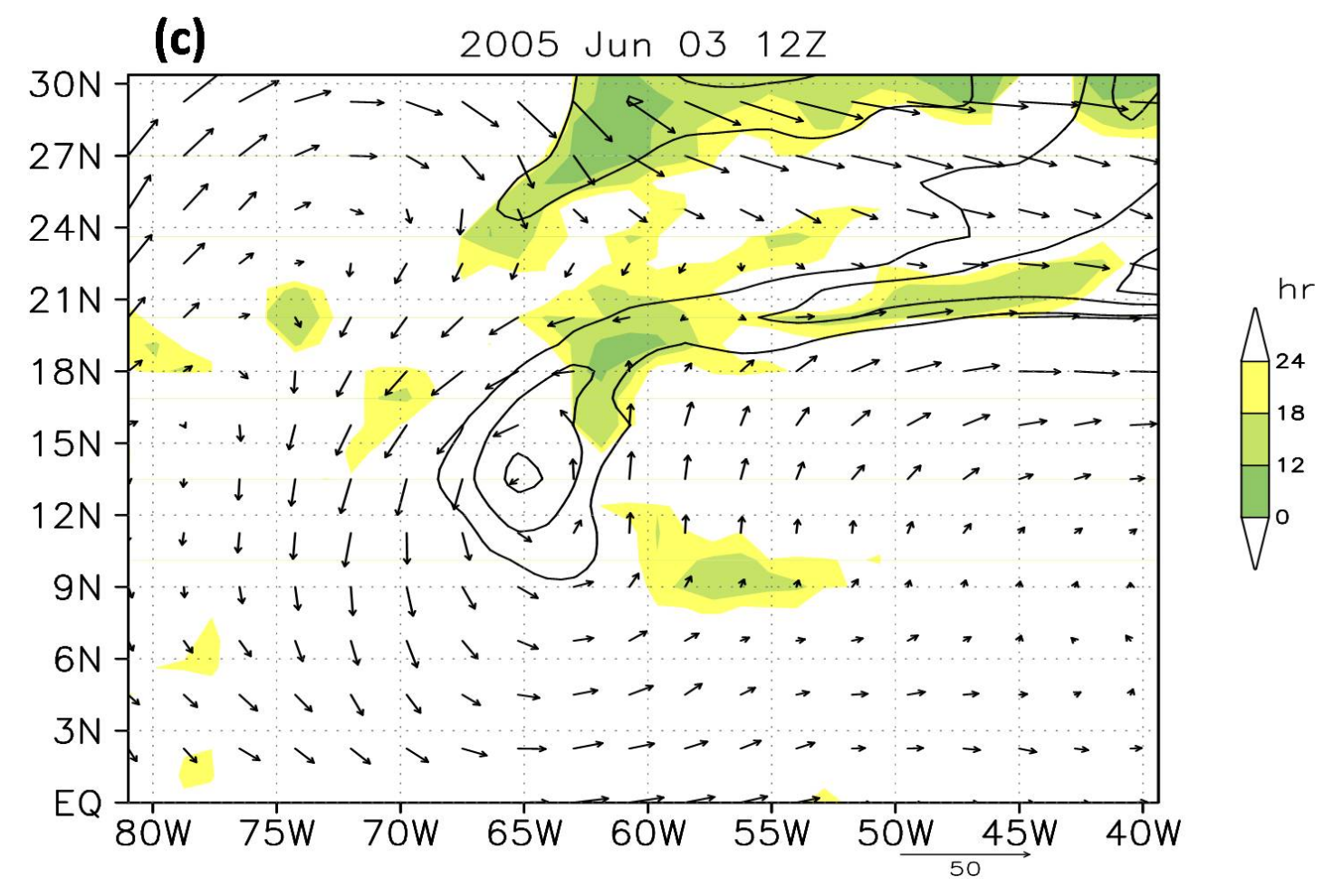

Figure 3: (Continued) 


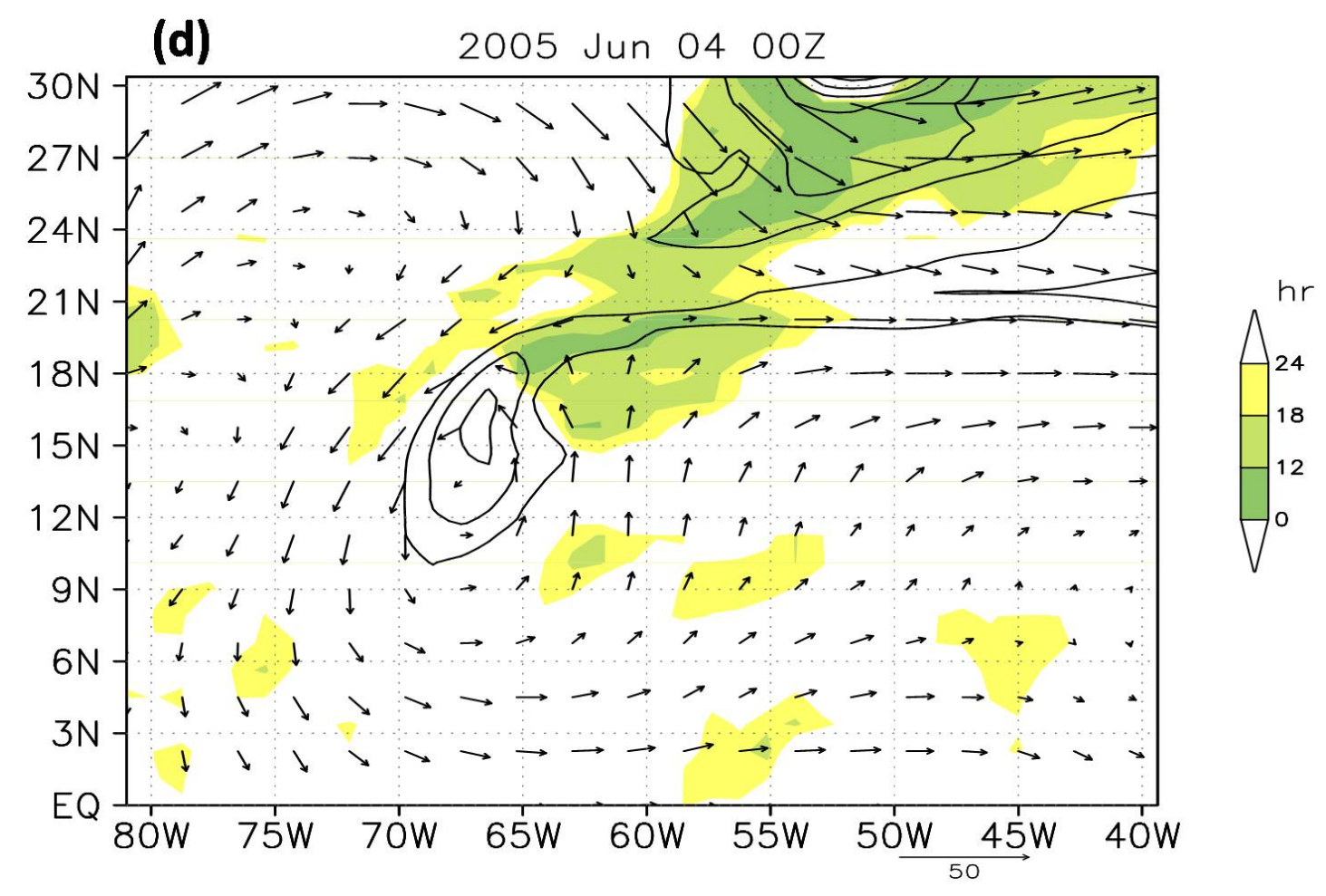

Figure 3: (Continued) 


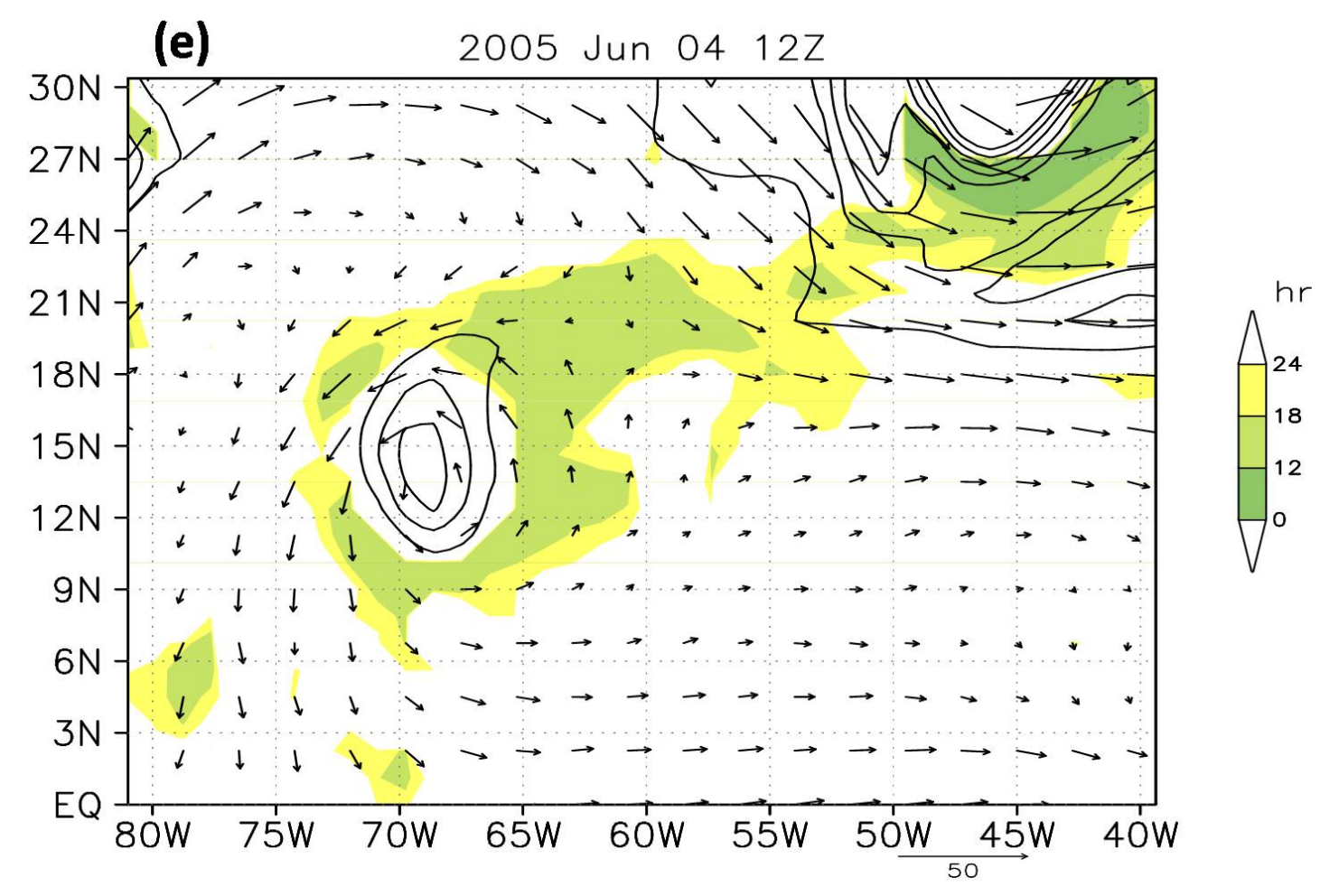

Figure 3: (Continued) 


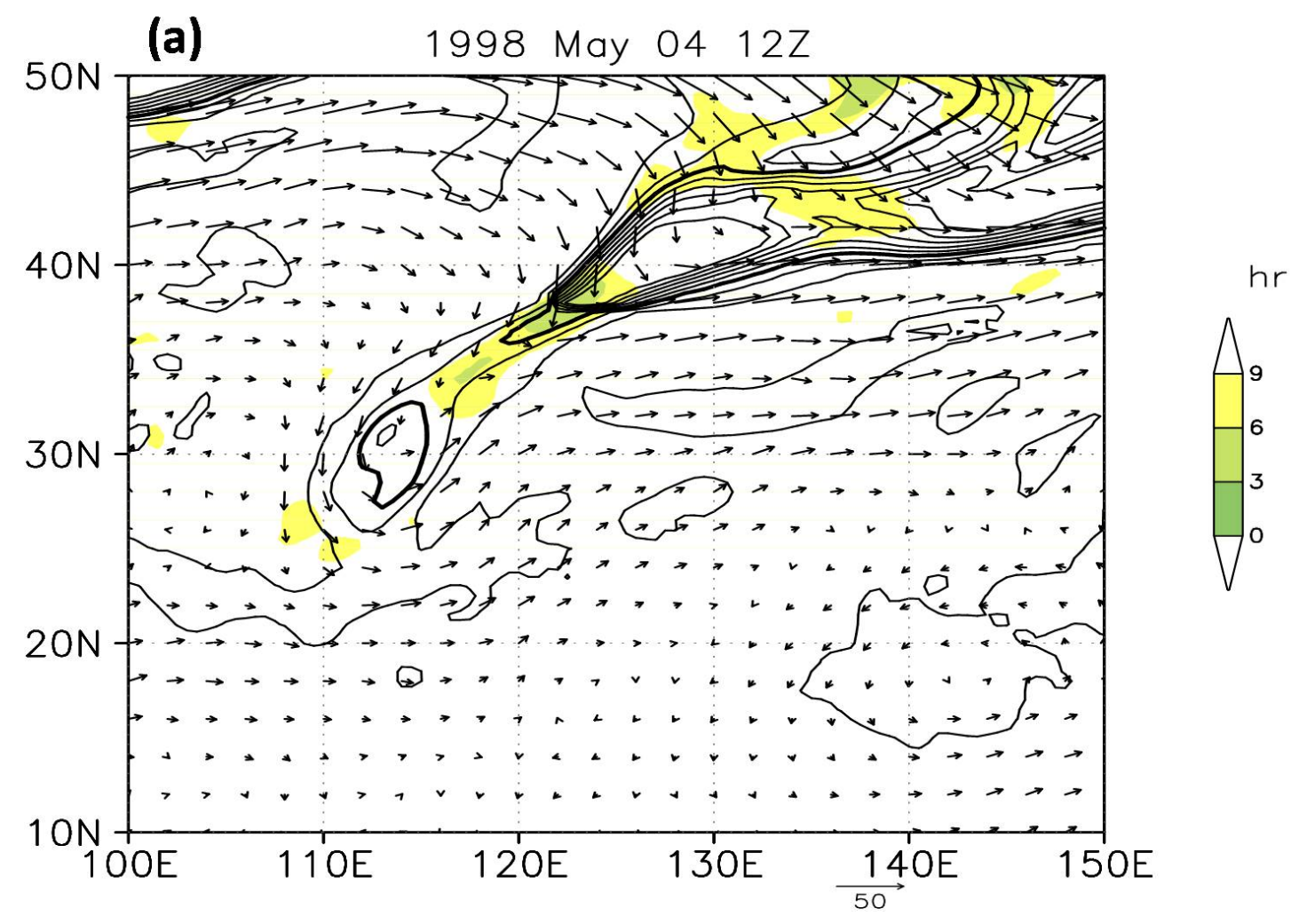

Figure 4: (a) The same as Fig. 2b but without the divergence term. (b) The same as Fig. 3c but without the divergence term. 


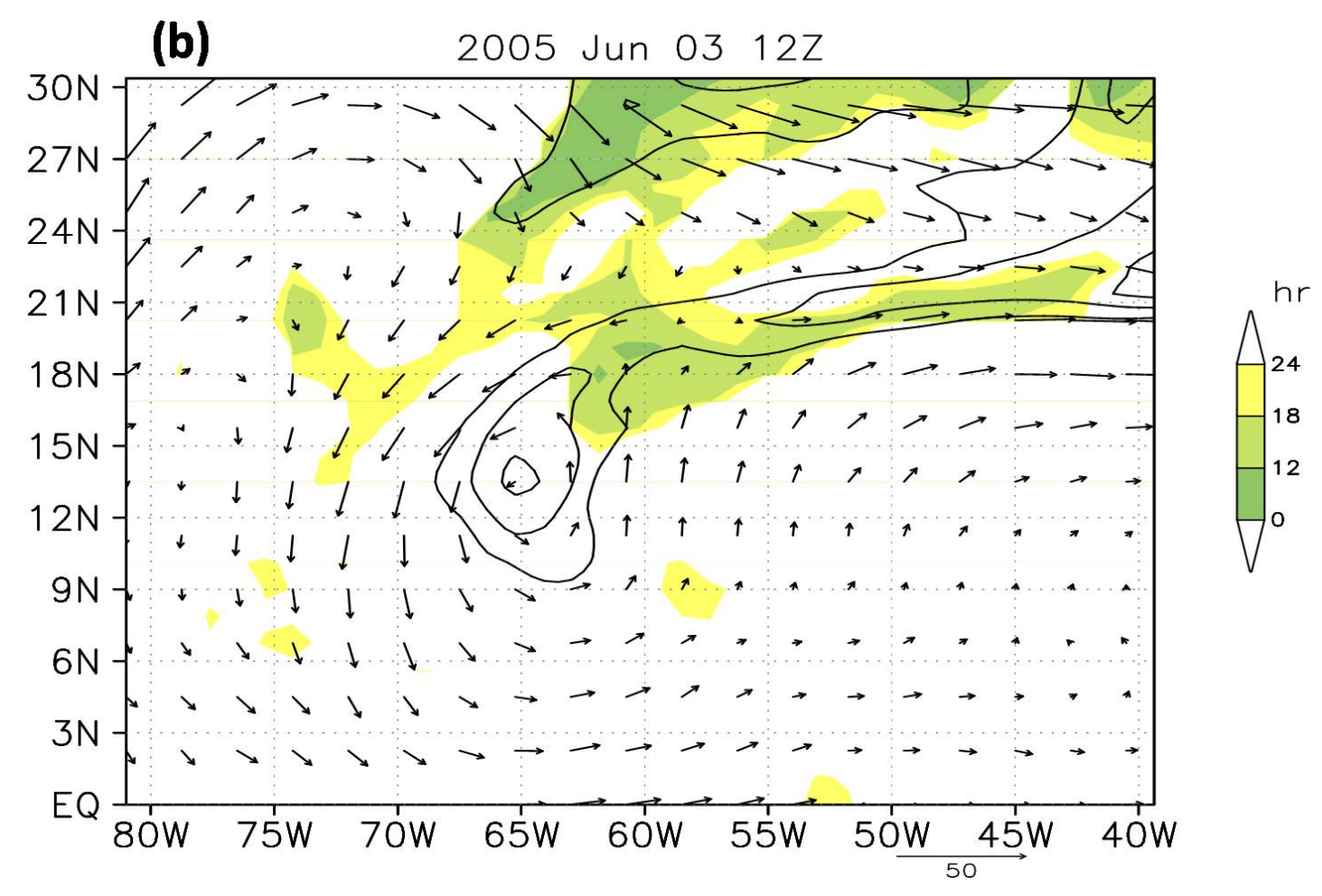

Figure 4: (Continued) 


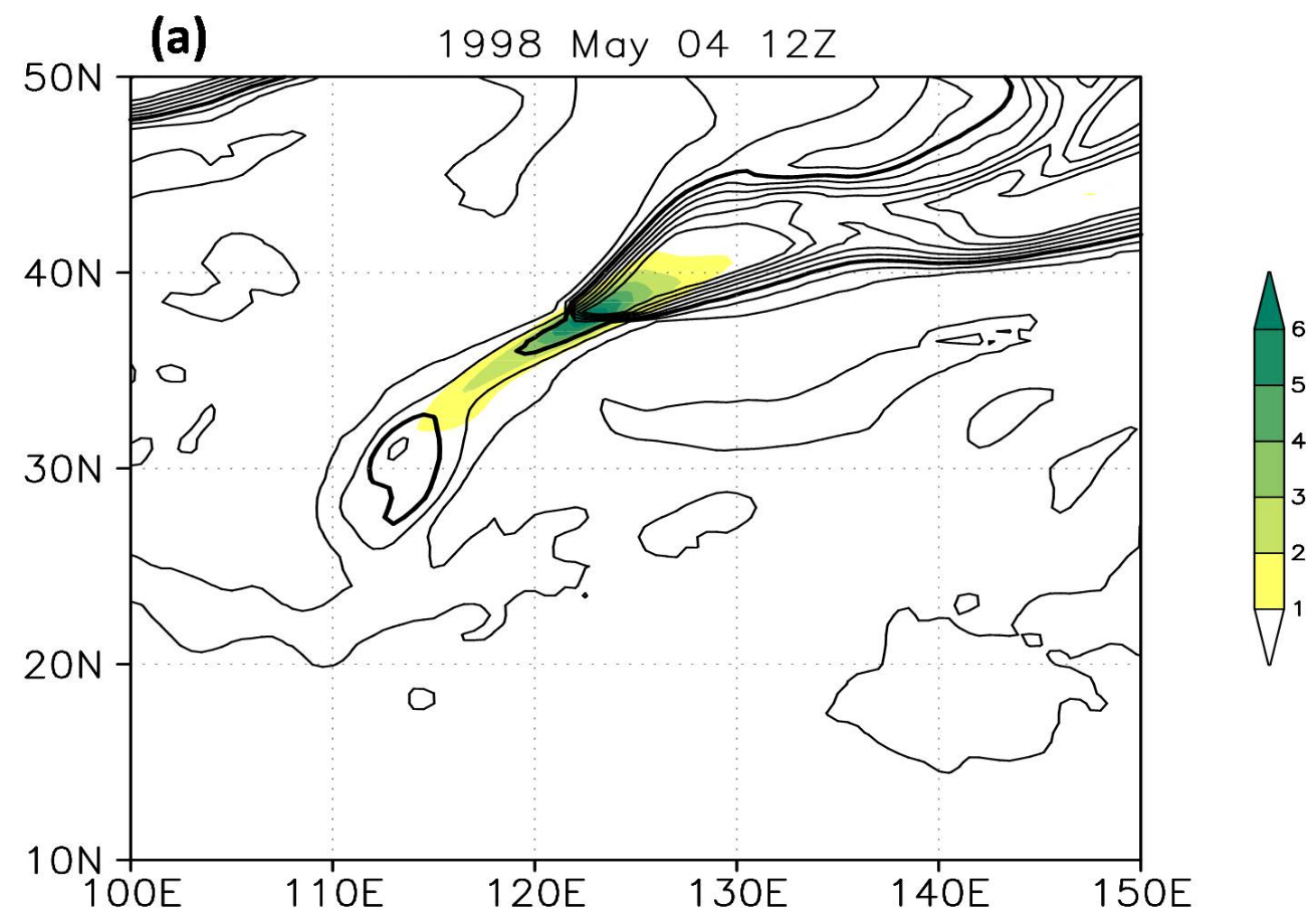

Figure 5: The potential vorticity (black contours, in PVU), and the deformation field (shading): (a) square of the stretching deformation in $10^{-8} \mathrm{~s}^{-2}$ for 4 May 199812 UTC; (b) square of the stretching deformation in $10^{-9} \mathrm{~s}^{-2}$ for 3 June $200512 \mathrm{UTC}$; (c) square of the shearing deformation in $10^{-9} \mathrm{~s}^{-2}$ for 3 June 200512 UTC. We only analyse the filamentation zone in the trough for the TUTT case. 


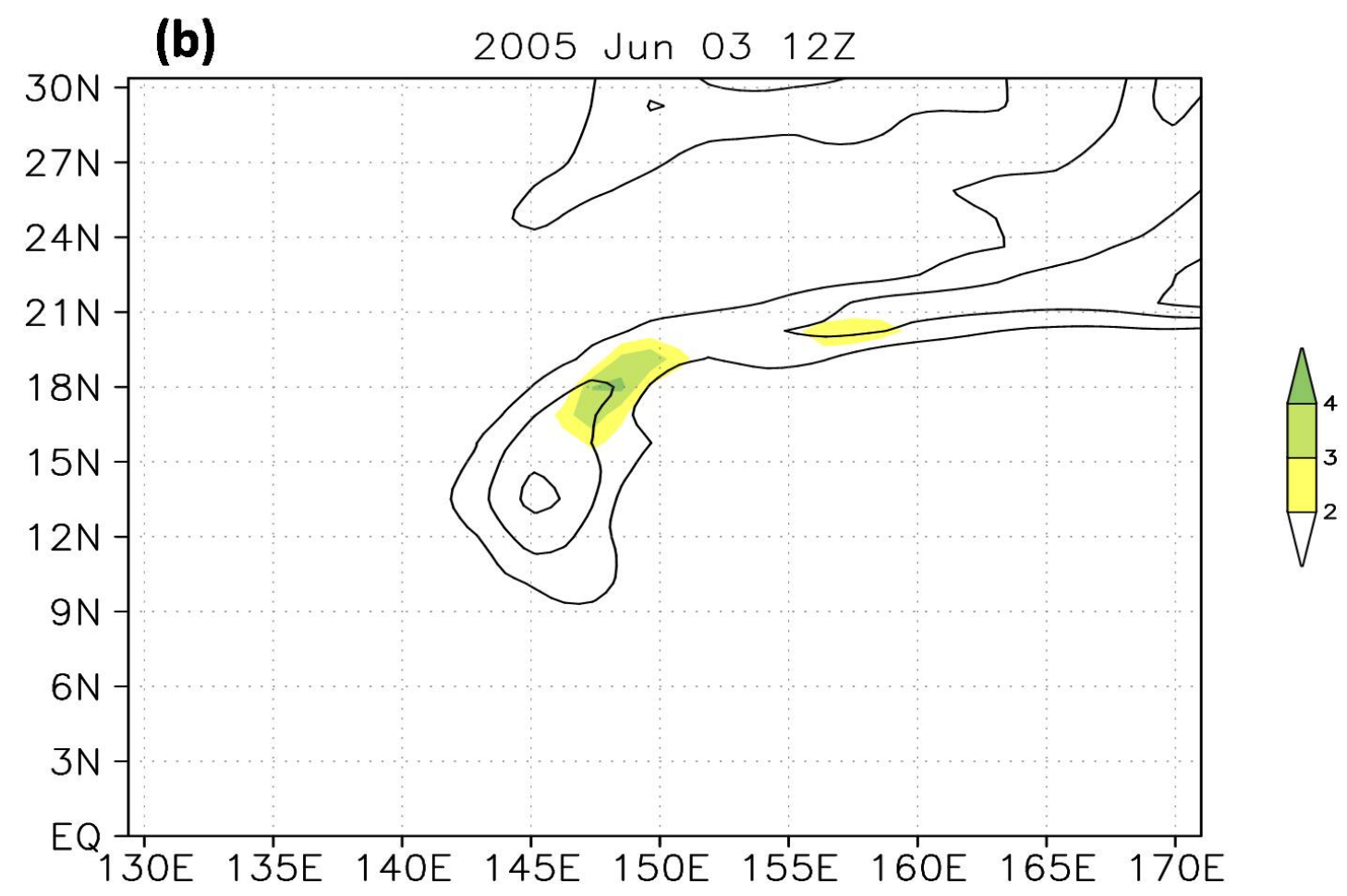

Figure 5: (Continued) 


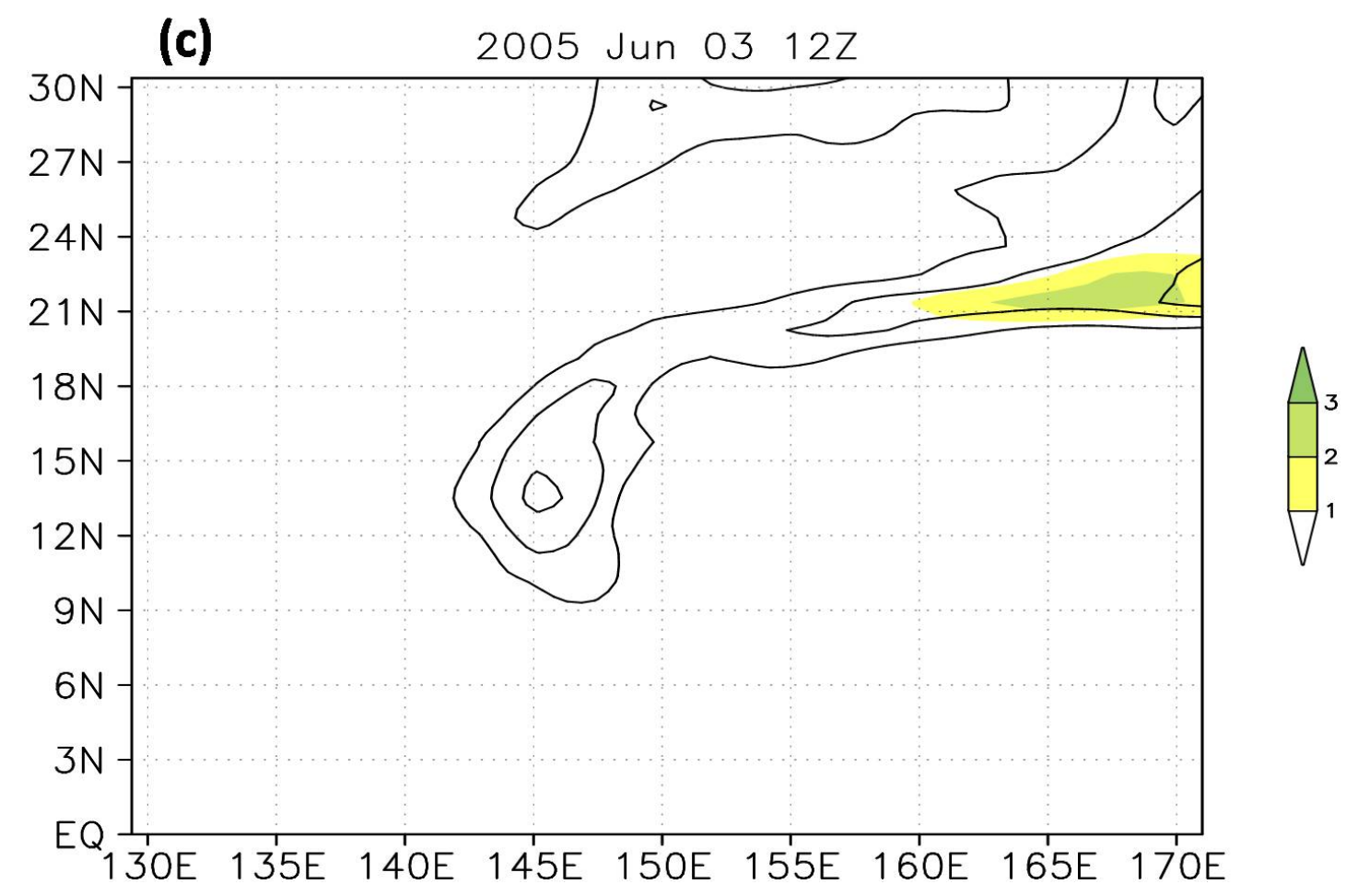

Figure 5: (Continued) 